\title{
Prevalência de pterígio no município de Botucatu - Estado de São Paulo, Brasil
}

\author{
Prevalence of pterygium in Botucatu city - São Paulo State, Brazil \\ Claudia Akemi Shiratori ${ }^{1}$, Josenylda Calixto de Barros ${ }^{2}$, Raquel de Matos lourenço ${ }^{2}$, Carlos Roberto Padovani ${ }^{3}$, Ricardo Cordeiro ${ }^{4}$, \\ Silvana Artioli SChellinI ${ }^{2}$
}

\section{RESUMO}

Objetivo: Avaliar a prevalência de pterígio no município de Botucatu - São Paulo, Brasil, por meio de amostra populacional.

Métodos: No ano de 2004 foi feito estudo de prevalência de alterações oftalmológicas na cidade de Botucatu - SP, por amostragem domiciliar aleatória, tendo sido avaliados 85,1\% (2.554 indivíduos) da amostra pretendida. Todos os participantes do estudo foram submetidos a exame oftalmológico completo, sendo os dados obtidos submetidos à análise estatística.

Resultados: A prevalência de pterígio na população estudada foi de 8,12\% $(7,0 \%<\mathrm{IC}<9,2 \%)$, sendo mais frequente em homens (10,4\% homens $\mathrm{X} 6,5 \%$ mulheres $-8,5 \%<\mathrm{IC}<12,3 \%$ em homens e $5,1 \%<\mathrm{IC}<7,8 \%$ em mulheres). A média de idade dos portadores de pterígio foi de 49,6 \pm 14,9 anos, havendo $32,18 \%$ indivíduos da amostra na faixa etária entre os 40 e 50 anos de idade. Conclusão: A prevalência do pterígio na cidade de Botucatu é de $8,12 \%$, sendo a lesão mais prevalente em homens, entre 40 e 50 anos de idade.

Descritores: Pterígio/epidemiologia; Amostragem; Intervalos de confiança; Brasil

\begin{abstract}
Purpose: To evaluate the prevalence of pterygium in a population-based sample at Botucatu City - São Paulo State, Brazil.

Methods: A population-based cross-sectional study with randomized clustered sampling of households was conducted in the urban area of the Botucatu City - São Paulo State, Brazil and $85.1 \%$ of the intended sample was evaluated. All participants were submitted to ophthalmologic examination and the data were statistically analyzed.

Results: The prevalence of pterygium lesion in Botucatu City was 8.12\% (7.0\% $<\mathrm{Cl}<9.2 \%)$, affecting mainly males $(10.4 \%$ males $X 6.5 \%$ females $-8.5 \%<\mathrm{Cl}$ $<12.3 \%$ for males and $5.1 \%<\mathrm{Cl}<7.8 \%$ for females) with $49.6 \pm 14.9$ years old in average; $32.18 \%$ of the pterygium carriers aged between 40 and 50 years. Conclusions: The prevalence of pterygium at Botucatu is $8.12 \%$, affecting most frequently 40-50 year-old males.
\end{abstract}

Keywords: Pterygium/epidemiology; Sampling studies; Confidence intervals; Brazil

\section{INTRODUÇÃO}

0 pterígio acomete indivíduos que habitam principalmente países de clima equatorial e tropical, localizados próximo ao equador e que trabalham expostos ao sol ${ }^{(1)}$ Estudo sobre a distribuição mundial desta afecção mostrou relação direta entre a taxa de prevalência e a proximidade da linha do equador, ocorrendo preferencialmente entre as latitudes $37^{\circ}$ Norte e Sul(2).

Em regiões de clima temperado, a prevalência é de cerca de $2 \%$, enquanto em regiões tropicais, varia entre o mínimo de $6 \%$, até mais de $20 \%{ }^{(3)}$. O Brasil, país de localização geográfica próxima à linha do equador, apresenta alta prevalência de pterígio $^{(4)}$.

Estudos brasileiros mostraram acometimento de ambos os sexos, nas mesmas proporções ${ }^{(4-5)}$. Entretanto, as avaliações

\footnotetext{
Trabalho realizado na Universidade Estadual Paulista "Julio de Mesquita Filho" - UNESP Botucatu (SP), Brasil.

Médica, Faculdade de Medicina de Botucatu, Universidade Estadual Paulista "Júlio de Mesquita Filho" - UNESP - Botucatu (SP), Brasil.

2 Médica, Departamento de Oftalmologia, Otorrinolaringologia e Cirurgia de Cabeça e PescoMédica, Departamento de Oftalmologia, Otorrinolaringologia e Cirurgia de Cabeça e PescoFilho" - UNESP - Botucatu (SP), Brasil.

Estatístico, Instituto de Biociências, Departamento de Bioestatística, Universidade Estadual Paulista "Julio de Mesquita Filho" - UNESP - Botucatu (SP), Brasil.

Médico, Faculdade de Ciências Médicas, Universidade Estadual de Campinas - UNICAMP - Campinas (SP), Brasil.

Endereço para correspondência: Silvana Artioli Schellini. Dep. OFT/ORL/CCP - Faculdade de Medicina de Botucatu - UNESP - Botucatu (SP) - CEP 18618-000

E-mail: sartioli@fmb.unesp.br

Recebido para publicação em 14.02.2009

Última versão recebida em 16.06.2010

Aprovação em 05.07.2010
}

geralmente são feitas em amostras de portadores submetidos à cirurgia, ou que procuram serviços oftalmológicos com queixas referentes à lesão ${ }^{(5-6)}$, portanto não refletem o real comportamento do pterígio dentro da população.

Portanto, considerando que os estudos populacionais devem mostrar como o pterígio realmente se distribui na população, além da observação da elevada ocorrência de pterígio no território brasileiro, foi desenvolvido este estudo, cujo objetivo foi avaliar a prevalência do pterígio na população geral.

\section{MÉTODOS}

Foi realizado estudo tranversal, de base populacional, realizado na cidade de Botucatu, São Paulo. Os dados observados fazem parte do Projeto de Prevenção da Cegueira da Faculdade de Medicina de Botucatu - UNESP. Foram considerados elegíveis para participar do estudo todos os indivíduos residentes no município no período de coleta dos dados, que ocorreu de agosto a dezembro de 2004.

A amostra foi domiciliar, aleatória, entre pessoas residentes na cidade de Botucatu. O critério de seleção do domicílio foi feito a partir de dados do último Censo Demográfico do IBGE, selecionando-se a primeira casa dos Setores Censitários e sistematicamente a sexta casa a partir daquela, do lado direito da rua, escolhido por sorteio. Caso a sexta casa estivesse desabitada, a primeira casa à direita da sorteada era escolhida. Se novamente a pesquisa resultasse negativa, a primeira casa da esquerda seria a escolhida e assim, sucessivamente. Assim feito em todos os bairros, mesmo quando havia alto nível de recusa em participação, até atingir a meta do número de indivíduos representativos da amostra. Todos os habitantes do 
domicílio eram considerados elegíveis (independentemente da faixa etária) e eram convidados a participar da pesquisa por meio de convite impresso, com conteúdo explicativo e terminologia simples sobre a importância do estudo. Havendo concordância na participação, era feito um contato telefônico para o agendamento da consulta oftalmológica, realizada no Ambulatório de Oftalmologia da Faculdade de Medicina de Botucatu.

O Comitê de Ética em Pesquisa em Seres Humanos da Faculdade de Medicina de Botucatu avaliou e aprovou o protocolo deste estudo.

Segundo dados do IBGE - 1995, a cidade de Botucatu possui cerca de 120.000 habitantes. A amostra foi estimada em 3.000 participantes (para determinar a prevalência de deficiência visual de $2 \%$ com uma precisão de 0,5\% e nível de significância de 5\%), baseado em estudo prévio de prevalência que indicava uma taxa de $2 \%$ - $4 \%$ de deficiência visual em Botucatu $^{(7)}$. Foram avaliados 2.554 indivíduos, correspondendo a $85,1 \%$ do total da amostra.

Todos os participantes foram submetidos a exame oftalmológico completo, feito por oftalmologistas ou residentes de Oftalmologia, e constou de: exame externo, acuidade visual usando a tabela de Optotipos de Snellen iluminada, colocada a 5 metros, com ângulo visual de 1 grau, em olhos separados, com o uso de correção óptica, refratometria com esquiascopia, biomicroscopia, fundoscopia com oftalmoscopia direta e tonometria com tonômetro de não contato (CT-60 computerized tonometer, Topcon, Tokyo, Japan); em crianças abaixo de 5 anos de idade, não foi realizada a medida da acuidade visual nem a tonometria. Os dados obtidos foram registrados em prontuário eletrônico.

Foram considerados para o presente estudo todos os pacientes portadores de pterígio detectados pelo exame biomicroscópico. O pterígio foi definido como a presença de uma cunha de tecido de aspecto fibrovascular ${ }^{(8)}$, com crescimento sobre o limbo corneano e/ou córnea. Foram excluídos os pacientes portadores de lesões suspeitas de outras doenças, como cicatrizes conjuntivais, leucoplasias, lesões de aspecto actínico ou neoplásico.

Os dados obtidos foram submetidos à análise estatística, empregando-se métodos de estatística descritiva e estudandose o intervalo de confiança (IC) para a proporção de ocorrência.

\section{RESULTADOS}

Dentre a amostra de 2.554 indivíduos, foram observados 190 casos de portadores de pterígio, constituindo uma prevalência geral de $8,12 \%(p=0,0812)$ de pterígio entre os habitantes da cidade de Botucatu, com variação de 7,0\% até 9,2\%. Dos portadores da lesão, $6,5 \%(p=0,0646)$ eram mulheres $(5,1 \%<\mathrm{IC}$ $<7,7 \%)$ e $10,4 \%$ ( $p=0,1038)$ eram homens $(8,5 \%<\mathrm{IC}<12,3 \%)$.

A média de idade dos portadores de pterígio foi de $49,6 \pm$ 14,9 anos. Nas mulheres a média de idade foi de 50,2 $\pm 14,2$ anos e nos homens de 49,0 $\pm 15,4$ anos (Tabela 1).

A distribuição dos casos segundo a faixa etária e o sexo demonstrou maior prevalência na faixa etária de 40 a 50 anos (28 casos), com a ocorrência de somente um indivíduo entre 10 e 20 anos de idade, que foi do sexo masculino (Tabela 2).

\section{DISCUSSÃO}

Este é o primeiro estudo que aponta a prevalência do pterígio em uma população geral brasileira. Outros estudos analisaram a ocorrência do pterígio, contudo, os dados eram restritos a populações previamente selecionadas, pois basearam-se em amostras de conveniência: dados de unidades de Tabela 1. Medidas descritivas da idade dos portadores
de pterígio - Faculdade de Medicina de Botucatu UNESP, 2004

\begin{tabular}{lccc}
\hline Medida descritiva & Geral & Masculino & Feminino \\
\hline Valor mínimo & 18,0 & 18,0 & 20,0 \\
P5 & 25,2 & 23,2 & 26,8 \\
P10 & 29,0 & 28,4 & 30,6 \\
P25 & 39,0 & 37,0 & 41,0 \\
P50 & 48,0 & 48,0 & 48,0 \\
P75 & 59,0 & 59,0 & 60,0 \\
P90 & 71,1 & 72,2 & 70,0 \\
P95 & 76,0 & 76,0 & 75,0 \\
Valor máximo & 88,0 & 87,0 & 88,0 \\
Média & 49,6 & 49,0 & 50,2 \\
Desvio padrão & 14,9 & 15,4 & 14,2 \\
\hline
\end{tabular}

referência hospitalar (estudos retrospectivos baseados em dados de prontuário ou em estudos prospectivos de pacientes submetidos à cirurgia). Estes dados traduzem a ocorrência dos pterígios que levam a sintomas, que por sua vez, motivam os indivíduos portadores a referir-se aos serviços de atendimento oftalmológico. Muitas vezes, dada a sua relativa baixa morbidade, parte dos portadores de pterígio não se apresenta aos serviços especializados, de forma que os números que indicam a frequência do pterígio na população geral sejam subestimados quando se toma por base estudos realizados em instituições hospitalares ou serviços oftalmológicos.

Devido à ausência de estudos de prevalência prévios para o pterígio nesta população, o número de participantes da amostra foi determinada baseando-se em estudo prévio de prevalência, contudo, considerando outras doenças oculares e não o pterígio isoladamente ${ }^{(7)}$.

Para a cidade de Botucatu, constatou-se que o pterígio tem a prevalência de $8,12 \%$; valor significativamente inferior ao encontrado no Peru, que foi de $31,1 \%{ }^{(9)}$. No entanto, o estudo peruano foi realizado em uma população hospitalar, o que pode justificar a elevada prevalência. Em estudo nacional realizado em Hospitais de Salvador e Manaus, observou-se dados semeIhantes ${ }^{(5)}$. Outro estudo do tipo populacional, realizado na Indonésia, país com características climáticas semelhantes ao Brasil, encontrou prevalência de $10 \%{ }^{(10)}$, valor semelhante ao encontrado neste estudo. A influência da localização geográfica mais próxima da linha do equador, em que o clima quente favoreceria a ocorrência do pterígio, não é corroborada por estudos que observaram que na África ${ }^{(11)}$ e na Austrália(12) a prevalência na população foi muito mais baixa, da ordem de 1,10\%. Diante destes dados, a localização geográfica isoladamente não seria determinante para ocorrência de pterígio.

Em relação à idade, a prevalência de pterígio em nosso meio foi maior na faixa etária de 40 a 50 anos, concordando com diversos trabalhos que relatam aumento da prevalência desta lesão com a idade ${ }^{(9,11,13-14)}$. Outro dado que se confirma é a baixa prevalência em jovens, não tendo sido encontrado nenhum caso abaixo dos 10 anos de idade, e também entre idades avançadas. Isto poderia ser decorrente de efeitos da radiação ultravioleta, devido à correlação existente entre esta condição e o desenvolvimento do pterígio(15-16), em que se observou que a radiação solar teria efeitos nocivos cumulativos, principalmente quando a exposição ocorre desde jovens e persiste por duas ou três décadas ${ }^{(15,17)}$.

Com relação ao sexo, a ocorrência de pterígio foi maior entre os homens $(10,4 \%)$, corroborando com a literatura ${ }^{(1,12-13)}$. Entretanto, estudos brasileiros mostraram acometimento de ambos 
Tabela 2. Distribuição dos portadores de pterígio segundo faixa etária e sexo -

Faculdade de Medicina de Botucatu - UNESP, 2004

\begin{tabular}{lccccc}
\hline & \multicolumn{2}{c}{ Masculino } & & \multicolumn{2}{c}{ Feminino } \\
\cline { 2 - 3 } Faixa etária & F absoluta & F relativa (\%) & & F absoluta & F relativa (\%) \\
\hline $10-20$ & 1 & 0,97 & & 0 & 0,00 \\
$20-30$ & 10 & 9,71 & & 8 & 9,20 \\
$30-40$ & 20 & 19,42 & & 10 & 11,50 \\
$40-50$ & 28 & 27,18 & 28 & 32,18 \\
$50-60$ & 20 & 19,42 & 19 & 21,84 \\
$60-70$ & 12 & 11,65 & & 12 & 13,79 \\
$70-80$ & 9 & 8,74 & & 9 & 10,34 \\
$80-90$ & 3 & 2,91 & 1 & 1,15 \\
Total & 103 & 100,00 & 87 & 100,00 \\
\hline
\end{tabular}

$F=$ frequência

os sexos, nas mesmas proporções ${ }^{(4)}$, ou predomínio no sexo feminino ${ }^{(18)}$. Para outros ${ }^{(5,9)}$, não há diferença significativa entre os sexos. Estas observações sugerem que o fator sexo não é determinante na possibilidade de desenvolver a lesão, dessa forma, outros fatores estariam envolvidos, como a exposição a outras variáveis que possam ser nocivas para a superfície ocular. O predomínio num determinado sexo pode estar influenciado pelo método empregado para a obtenção dos dados. Numa amostra hospitalar, composta de indivíduos submetidos à cirurgia de ressecção do pterígio, o predomínio de mulheres pode estar relacionado ao fator estético, que motivaria a maior procura por tratamento ${ }^{(18)}$.

No presente estudo, não foi avaliada a atividade laboral dos indivíduos estudados, não sendo possível estudar a relação entre o tipo atividade e a ocorrência do pterígio. A atividade laboral é uma variável mutável ao longo do período de vida. Um indivíduo que no momento da coleta de dados não exerça uma atividade exposta à radiação solar, pode ter exercido outra ocupação anterior com forte exposição.

O pterígio, sendo condição multifatorial, torna-se assunto de complexo entendimento, sendo necessário abordar os diversos fatores que influenciam a sua etiogenia e evolução, ainda carente de métodos para mensurar de forma adequada cada um desses fatores, sendo uma limitação dos estudos transversais.

\section{CONCLUSÃO}

Portanto, a prevalência do pterígio na cidade de Botucatu é de $8,12 \%$, sendo a lesão mais prevalente em homens, entre 40 e 50 anos de idade. $O$ estudo da prevalência na população geral é importante para compreender o real comportamento do pterígio, sem submeter a dados superestimados obtidos em centros de referência em oftalmologia.

Outros estudos prospectivos, isolando cada uma das variáveis que influenciam o pterígio seriam necessários para avaliar a sua ocorrência e morbidade.

\section{REFERÊNCIAS}

1. Saw SM, Banerjee K, Tan D. Risk factors for the development of pterygium in Singapore: a hospital based case-control study. Acta Ophthalmol. 2000;78(2): 216-20.

2. Cameron ME. Geographic distribution of pterygium. Trans Ophthalmol Soc Aust. 1962:22:67-81.

3. Cornand G. [Pterygium. Course and treatment]. J Fr Ophtalmol. 1990:13(1-2):33-45. Review.

4. Garrido C, Carvalho RC, Garrido Neto T, Magalhães LC, Cohen J, Cunha M, et al. Avaliação da acuidade visual e da ceratometria após a cirurgia do pterígio. Arq Bras Oftalmol. 1996;59(6):614-6.

5. Garrido Neto T, Garrido C, Carvalho RC, Lima HC. Estudo da frequencia de pterígio em Hospitais de Salvador e Manaus. Rev Bras Ofaltamol. 1996:55(9):683-6.

6. Schellini SA, Veloso CER, Lopes W, Padovani CR, Padovani CRP. Características de portadores de pterígio na região de Botucatu. Arq Bras Oftalmol. 2005;68(3): 291-4

7. Silva MRB, Schellini SA, Kamegasawa A, Heimbeck FJ, Carandina L. Levantamento de cegueira em Botucatu - prevalência e causas. Rev Bras Oftalmol. 1986; 45(1):18-23.

8. Alves MR, Vitor G. O tratamento do pterígio. Rev Bras Oftalmol. 2005;64(5):351-62. 9. Rojas JR, Malaga H. Pterygium in Lima, Peru. Ann Ophthalmol. 1986;18(4):147-9.

10. Gazzard G, Saw SM, Farook M, Koh D, Widjaja D, Chia SE, et al. Pterygium in Indonesia: prevalence, severity and risk factors. Br J Ophthalmol. 2002;86(12):1341-6.

11. Ebana Mvogo C, Bella-Hiag A, Ngosso A, Ellong A. Lê pterygion: aspects épidémiologiques cliniques et thérapeutiques a l'hôpital general de Douala. Méd d'Afrique Noire. 1997;44(5):290-2.

12. Wlodarczyk J, White $P$, Cockrum $P$, Taylor H. Pterygium in Australia: a cost of illness study. Clin Experiment Ophthalmol. 2001;29(6)370-5. Comment in: Clin Experiment Ophthalmol. 2002;30(4):312.

13. Tan D, Tang WY, Liu YP, Goh HS, Smith DR. Apoptosis and apoptosis related gene expression in normal conjunctiva and pterygium. Br J Ophthalmol. 2000; 84(2): 212-6.

14. Alves MR. Pterígio In: Alves MR. Conjuntiva cirúrgica. São Paulo: Roca; 1999. p.59-66

15. Mackenzie FD, Hirst LW, Battistutta D, Green A. Risk analysis in the development of pterygia. Ophthalmology. 1992;99(7):1056-61.

16. McCarty CA, Fu CL, Taylor HR. Epidemiology of pterygium in Victoria, Australia. $\mathrm{Br}$ J Ophthalmol. 2000;84(3):289-92

17. Tang FC, Chen SC, Lee HS, Lin WF, Chou MC, Lee MC. Relationship between pterygium/pinguecula and sunlight exposure among postmen in central Taiwan. Zonghua Yi Xue Za Zhi (Taipei). 1999;62(8):496-502.

18. Christov R, Forno EA, Campagna CM, Bechara SJ, José NK. Análise histopatológica de pterígios primários e recidivados. Rev Bras Oftalmol 1991;50(4):59-62. 